\title{
Estimation of the Respiratory Activity from Orthogonal ECG Leads
}

\author{
AM Bianchi, GD Pinna*, M Croce, MT La Rovere*, R Maestri*, E Locati**, S Cerutti \\ Dipartimento di Bioingegneria, Politecnico di Milano, Italy, * S. Maugeri Foundation, IRCCS \\ Montescano, Italy, **Department of Cardiology, University of Perugia, Italy
}

\begin{abstract}
In the present work a method is proposed for the estimation of the respiratory signal from the three ECG orthogonal leads.

The vectorcardiogram loop was evaluated in correspondence of each QRS complex (in a time window of $120 \mathrm{~ms}$ centred around the $R$ wave). The centre of gravity and the inertial axes of each loop were calculated. A set of 12 parameters (3 spatial coordinates of the centre of gravity and 3 directions of the 3 inertial axes) was obtained for each cardiac beat. The first principal component of such a set of data was the estimation of the respiratory activity.

The obtained signal was compared with the quantitative respiration recording and the crosscorrelation index $C$ was a measure of the goodness of the estimation. The mean value of $C$ was $0.9 \pm 0.26$, indicating a good matching between the recorded and the estimated signals.
\end{abstract}

\section{Introduction}

The analysis of many signals in association with the Holter recorded ECG, is now achieving great importance for the diagnosis of different pathologies affecting the cardio circulatory system. Among them the respiratory signal is of paramount relevance. In fact, anomalies and diseases of the respiratory activity (i.e. nocturnal apnoeas) have been proved to be a risk factor in different patient populations with, or without, diagnosis of cardiopathy [1]. The direct recording of the quantitative respiratory signals (airflow or air volumes) presents relevant practical problems mainly for the equipments that strongly interfere with the patient and thus are not particularly suitable for long duration recordings, such as ambulatory monitoring or sleep evaluations. On the other hand, it is well known that respiratory activity heavily affects many physiological parameters, thus, from this point of view, its estimation from other, more accessible, signals seems to be more feasible. In particular, in case of Holter clinical investigation, it is useful to estimate the respiratory signal from the modifications that it induces in the ECG. Usually respiratory related beat-to-beat modifications in the ECG morphology are considered as a disturbance and different methods have been proposed for their identification and reduction [2, 3]. In particular respiratory movements affect geometrical vectorcardiographic (VCG) characteristics in the 3D space representation. Such modifications can constitute the bases for an estimation of the respiratory signal [4].

\section{Methods}

It is well known that respiration induces different modifications on the ECG. Such variations are mainly related to: amplitude of the $\mathrm{R}$ peak, areas under the QRS complex, oscillations of the baseline, variations in the electrical cardiac axes, variability in the R-R interval. They can be differently quantified and related to respiration as reported in previous studies [5-8].

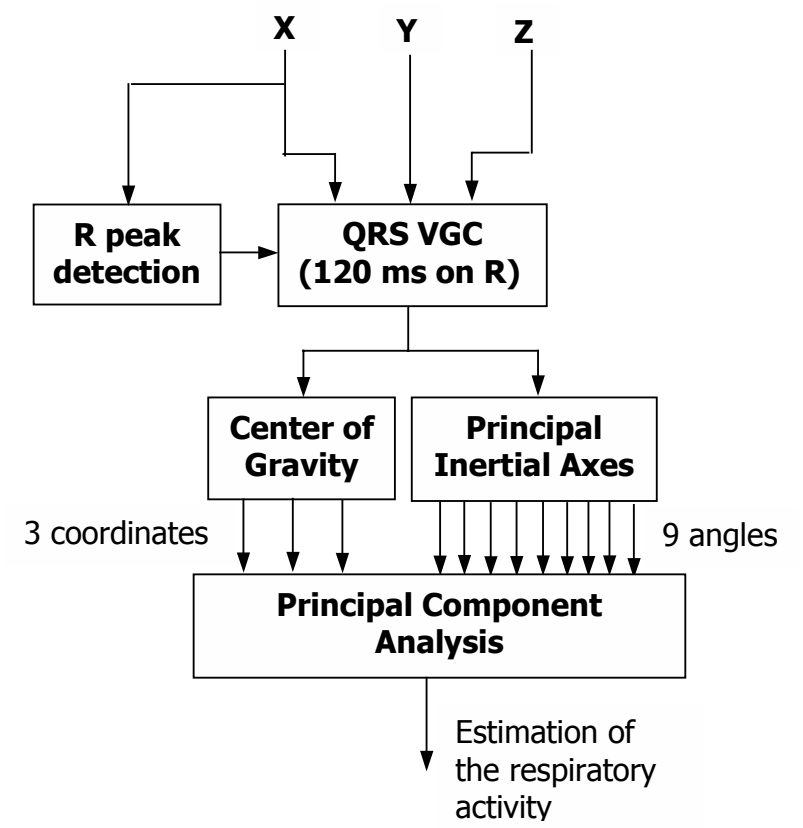

Figure 1 Block diagram of the procedure implemented for the estimation of the respiratory activity from three orthogonal ECG leads

In particular the presented method is based on the evaluation of Vectorcardiogram (VCG) loops and on the 
detection of the beat-to-beat variations of the loop geometric parameters in the 3D space. Each loop varies in shape, and in its distribution in the 3D space for different causes, among which the most relevant is the respiratory activity.

The steps of the complete procedure are summarized in Fig. 1.

The QRS complex and the R peak are firstly identified on the X lead through the Pan-Tomkins algorithm [9]. A time window of $120 \mathrm{~ms}$ including the QRS complex (60 $\mathrm{ms}$ before and $60 \mathrm{~ms}$ after the $\mathrm{R}$ peak) is considered for the analysis and a QRS loop was constructed for each beat. Each loop is viewed as a cluster of points in the $3 \mathrm{D}$ space, with its own inertial properties. Thus for each cluster (for each loop) the centre of gravity was calculated, that provides three parameters $\left(x_{c}, y_{c}, z_{c}\right.$ coordinates) related to each cardiac beat. The inertial axes, in the space, for each loop were also obtained. Each inertial axis is completely identified in the space through the three angles formed with the axis of the reference system: we obtain 9 cosines for each loop and then for each beat. Globally we obtain 12 parameters for each cardiac beat, each of them is affected by respiration activity and provides information on respiration.

Fig.2 shows an example of the extracted parameters. Panel $a, b$ and $c$ show the coordinates $(x, y$ and $z$ respectively) of the centre of gravity. Panels $d$, $e$ and $f$ are related to the first inertial axes. Panels $g, h$ and $i$ are related to the second inertial axes, and finally panels $l, m$ and $n$ are related to the third one. They represent the cosines of the angles between each inertial axes and the axis of the reference system, $x, y$ and $z$, respectively. Panel $o$ is the measure of the respiratory volume obtained integrating the measured airflow. The 12 estimated parameters are not independent and are strongly related to the respiration.

The method of the principal component analysis (PCA) allows to identify the linearly independent sources $\mathrm{S}_{\mathrm{k}}$ contributing in the genesis of the signals. Respiration is hypothesized to be the one strongly affecting the whole set of parameters, thus the highest igenvalue (corresponding to the source that causes the highest variance in the signals) is supposed to be related to the respiration and is the final estimation of the respiratory activity.

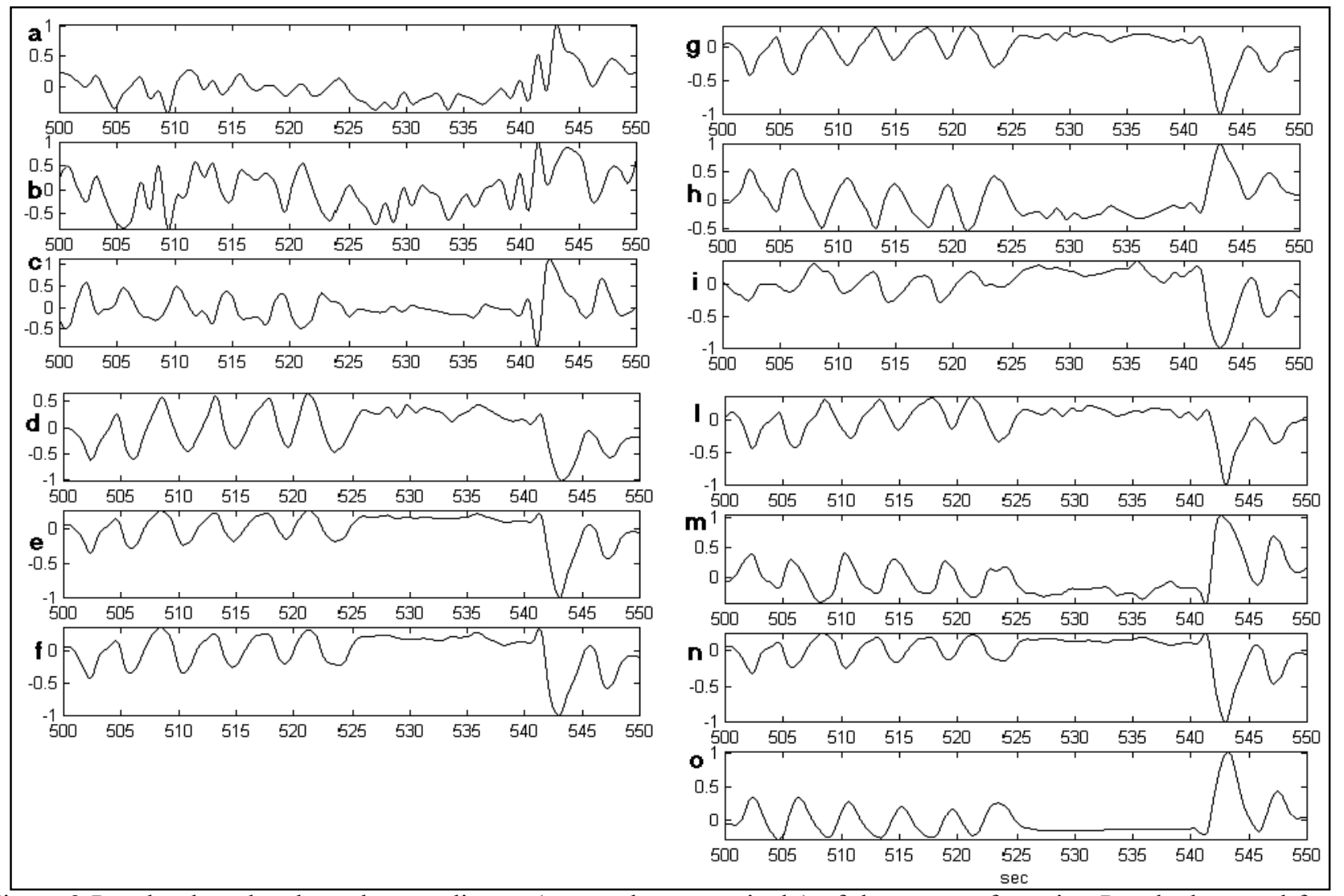

Figure 2 Panel $\mathrm{a}, \mathrm{b}$ and $\mathrm{c}$ show the coordinates (x, y and $\mathrm{z}$ respectively) of the centre of gravity. Panels d,e e and $\mathrm{f}$ are related to the first inertial axis. Panels $\mathrm{g}, \mathrm{h}$ and $\mathrm{i}$ are related to the second inertial axes. Panels $\mathrm{l}, \mathrm{m}$ and $\mathrm{n}$ are related to the third inertial axis. They represent the cosines of the angles between each inertial axes and the axes of the reference system. Panel o is the respiratory air volume obtained integrating the measured airflow. 
In order to test the performances of the procedure, it was applied to ECG recordings, during which also a quantitative measure of the respiration was obtained.

\section{Experimental protocol}

The signals for the technical evaluation of the algorithm were recorded in the Neurovegetative Laboratory of the S. Maugeri Foundation, IRCCS Scientific Institute in Montescano. 4 normal subjects underwent a 20 min recording of the ECG signal through three orthogonal leads, $\mathrm{X}, \mathrm{Y}, \mathrm{Z}$ and the respiratory airflow through Pneumotac device.

During the recording session the epochs listed in Tab.1 were considered:

Table 1. Epochs during the experimental protocol.

\begin{tabular}{ll}
\hline Subject Position & Time Duration \\
\hline Supine & $3 \mathrm{~min}$ \\
Simulated apneas & $5,10,15,20 \mathrm{sec}$ \\
Lying on the right side & $2 \mathrm{~min}$ \\
Lying on the left side & $2 \mathrm{~min}$ \\
Sitting & $2 \mathrm{~min}$ \\
Standing & $3 \mathrm{~min}$ \\
Step & $2 \mathrm{~min}$ \\
\hline
\end{tabular}

In patrticular each subject simulated 4 apneas of 5,10 , 15 and $20 \mathrm{~s}$. Between them the subjects breathed normally for a period of $1 \mathrm{~min}$. The signals were properly filtered and sampled at $200 \mathrm{~Hz}$. A measure of the air volume was obtained integrating the measured airflow. This signal was considered for the following comparison with the estimated respiration and is referred in the paper as real respiration signal. The method described in section 2 was applied. After the inertial parameters were obtained, the PCA was repeatedly calculated after segmentation of the traces in time windows of 20,40, 50, $80,100,160,200,400,800 \mathrm{sec}$. This was done for testing the performances of the procedure with different settings of the parameters. A beat-to-beat estimation of the respiratory signal was obtained. The trace was then interpolated in order to provide a $200 \mathrm{~Hz}$ sampled signal for a better visual comparison with the quantitative measure of respiration, as shown in Fig. 2 and in Fig 3. A correlation index $\mathrm{C}$ between the real and the estimated signals, after normalization, in time and in frequency domains, was calculated as a measure of the goodness of the estimation both in time and in frequency domain.

\section{Results}

An example of the obtained estimation is plotted in Fig.3 that shows the real respiration signal (continuous line) and the estimated one (dashed line) in correspondence of a simulated apnea. The good visual matching is also confirmed by the quantitative comparison performed through the $\mathrm{C}$ index.

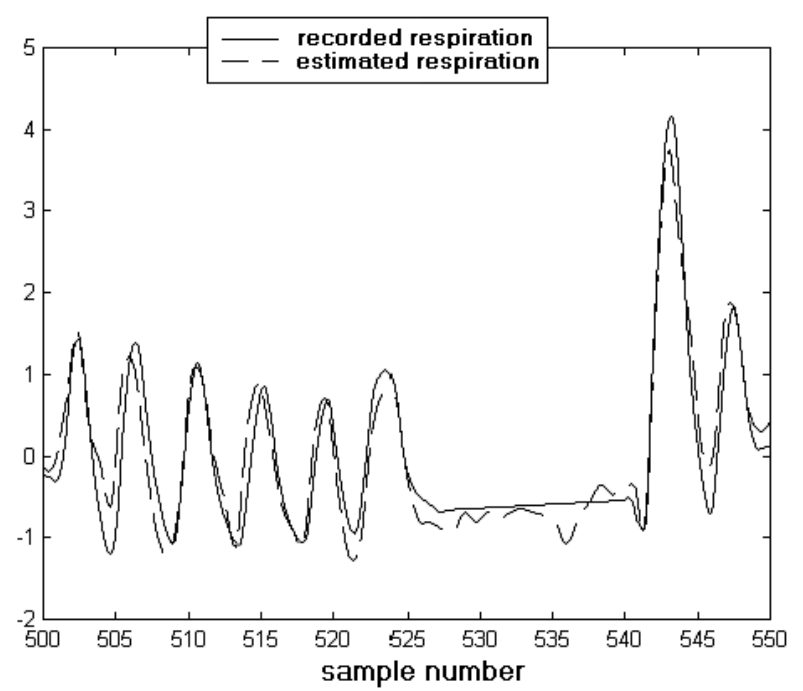

Figure 3 The real respiration signal obtained integrating the measured airflow is represented with a continuous line, while the dashed line represents the estimated respiration signal.

The mean value of $\mathrm{C}$, in frequency domain, on analysis windows of $20 \mathrm{~s}$, was $0.92 \pm 0.0 .4$, indicating a good matching between the recorded and the estimated signals. The same parameter was also evaluated considering different window lengths and the results are shown in Fig. 4. For window length below $80 \mathrm{~s}$, we obtained similar results; when the window length is 80 or $100 \mathrm{~s}$ we observed a slight and not significant decrease in the performances of the algorithm; for window length of 160,200 and $400 \mathrm{~s}$ the mean value of $\mathrm{C}$ decreases and the standard deviation increases, indicating high variability in the performances. For window length of $800 \mathrm{~s}$ the performances of the procedure are similar to the ones obtained for windows of 80 and $100 \mathrm{~s}$.

A second important aspect, that was considered in the evaluation of the algorithm, is its performance related to the subject's position. It is well known that the electrical cardiac axes, and then the vectorcardiogram, can be modified by the body position and that it is strongly related to body movements different from respiration. Fig. 5 shows the $\mathrm{C}$ index in mean value \pm standard deviation over the considered population in function of the subject's posture. In the present evaluation the window length for the analysis was $50 \mathrm{~s}$.

Good performances of the procedure are related to the lying position, with an improvement when the subjects were on the left side and a worsening when the subjects 
were lying on the back.

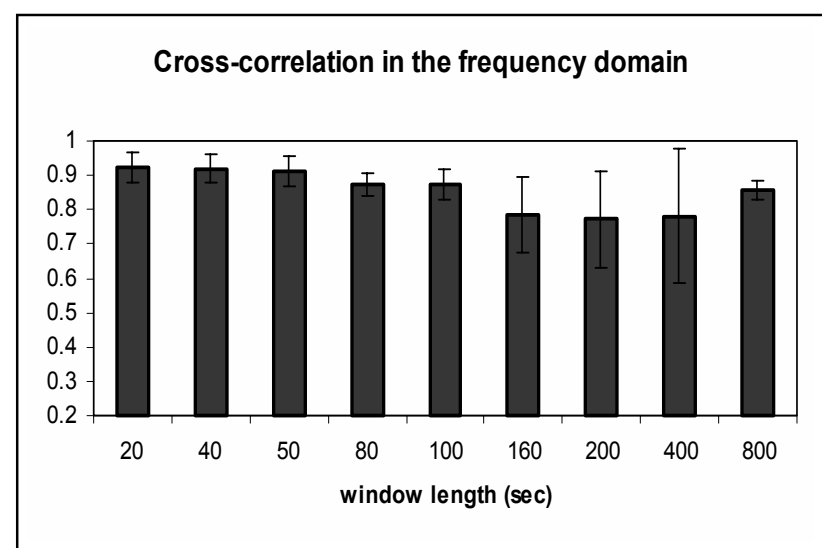

Figure 4 Correlation coefficient $\mathrm{C}$ between estimated and real respiration signals in function of the time window length on which the estimation was performed. The mean value \pm standard deviation is represented.

Also in standing position we obtained a good estimation with a slight decreasing during stepping. The worst result is related to the condition of simulated apneas which shows a mean value of $\mathrm{C}$ below 0.9 , with a high standard deviation.

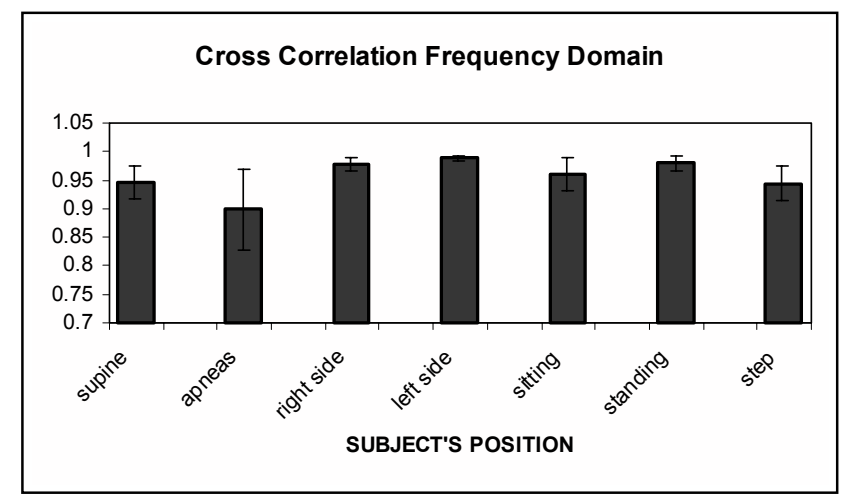

Figure 5 Correlation coefficient $\mathrm{C}$ between estimated and real respiration signals in function of the subject's position, expressed as mean value \pm standard deviation over the examined population.

\section{Discussion and conclusion}

The presented method provided suitable estimation of the respiratory activity from ECG orthogonal leads. Its performances were tested on real signals from 4 normal subjects in different conditions and with different settings of the algorithm parameters and always a good matching between the real respiration and the estimated traces was obtained. A further investigation is now required from a clinical point of view, in order to verify whether an observation performed on real or on estimated respiration can lead to comparable diagnosis of the pathology (for example sleep apnea syndrome).

A possible application is in a more complete analysis of the Holter recordings and for a continuous monitoring of the respiration in ambulatory patients. The adjunctive information have a relevant impact from a clinical point of view, for a more complete diagnosis of the cardiovascular pathologies. The future is towards the development of "intelligent devices" with the processing module integrated in the sensors, for the on-line extraction of the relevant parameters, which will be transmitted to a clinical expert or to a recording system or may be used for the real-time identification of emergency events. The aim is to provide a global view of the patient status and to improve the quality of his/her life.

\section{Acknowledgements}

The paper was partially supported by an ELA Medical grant inside the DRESH (Development Research Electocardiographic Signal Holter) project. In particular, the authors wish to thank ing. Andrea Pinciroli for his help in the organization of the work.

\section{References}

[1] Nieto FJ, Young TB, Lind BK, Shahae E et al. Association of sleep-disordered breathing, sleep apnea, and hypertensionin a large community-based study. JAMA 2000; 283:1829-36.

[2] Sornmo L. Vectorcardiographic loop alignment and morphologic beat-to-beat variability. IEEE Trans. on Biom Eng. 1998; 45:1401-13.

[3] Pellegrini A, Pinciroli F, Rossi R, Vergani L. Virtual leads for ambulatory ECG monitoring: remarks on the persistency of their direction. Comp. And Biom. Res. 1985; 18:62-78.

[4] Pinciroli F, Rossi R, Vergani L, Carnevali S, Mantero S, Parigi O. Remarks and experiments on the construction of respiratory waveforms from electrocardiographic tracings. Comp. Biom. Res. 1985; 19: 391-409.

[5] Moody GB, Roger GM, Zoccola A, Mantero S. Derivation of respiratory signals from multilead ECGs. Computers in Cardiology 1985; 12:113-6.

[6] Travaglini A, Lamberti C, De Bie J, Ferri M. Respiratory signal derived from eight-lead ECG. Computers in Cardiology 1998;25:65-8.

[7] Leanderson S, Laguna P, Sornmo L. Estimation of the respiratory frequency usung spatial information in the VCG. Med. Eng. \& Phys. 2003; 25:501-7.

[8] de Chazal P, Heneghan C, Sheridan E, Reilly R, Nolan P, O'Malley M. Automated processing of the single-lead electrocardiogram for the detection of obstructive sleep apnoea. IEEE Trans. Biom. Eng. 2003; 50:686-96.

[9] Pan J, Tomkins WJ. A real-time QRS detection algorithm. IEEE Trans. Biom. Eng. 1985;32:230-6.

Address for correspondence.

Anna M. Bianchi

Dip.di Bioingegneria, Politecnico di Milano, piazza Leonardo da Vinci 32, 20133 Milano, Italy,

Annamaria.bianchi@polimi.it 